\title{
The application of Industrial machine vision detection and location technology in car key assembly equipment
}

\author{
Ye $\mathrm{Li}^{1, \mathrm{a}}$, Luo Bin ${ }^{2, \mathrm{~b}}$, Yang Janghua ${ }^{3, \mathrm{c}}$
}

${ }^{1}$ Chongqing Institute of Mechanical \& Electrical Engineering, Long Xi Song Pai Road 98 Hao , Yu Bei , Chongqing , 401147, China

${ }^{2}$ Chongqing Institute of Mechanical \& Electrical Engineering, Long Xi Song Pai Road $98 \mathrm{HaO}, \mathrm{Yu}$ Bei , Chongqing , 401147, China

${ }^{3}$ Chongqing Institute of Mechanical \& Electrical Engineering, Long Xi Song Pai Road $98 \mathrm{HaO}, \mathrm{Yu}$ Bei , Chongqing , 401147, China a,b,c624525078@qq.com

\begin{abstract}
Keywords: car keys assembly, automatic assembly, visual positioning technology
\end{abstract}
Abstract: This paper introduces the auto wireless automatic assembly system, an automatic assembly equipment. Present domestic car keys assembly way is mainly manual assembly. Manual assembly production efficiency is low, the intensity of labor is big, high cost. In order to change this way of backward manual assembly car keys, and adapt to the demands of the rapid development of China's automobile product quantity. Our enterprise use visual positioning technology independently developed car keys automatic assembly system. And it will be used in actual production, improving the efficiency of the lock core assembly, reducing the production cost.

\section{Introduction}

the only character recognition on the ontology of car keys is a key process in car keys assembly, and directly affects the assembly efficiency and the yield of keys. At present, the widely used method is artificial recognition and sensor machinery. Artificial recognition method is low efficiency, poor reliability and unable to meet the requirements for industrialized mass production. Mechanical identification has some disadvantages such as recognition accuracy not high, the low degree of automation and mechanical loss. This article innovation put forward a kind of automatic identification method based on machine vision measurement technology, machine vision measuring system is accurately fast, stable and reliable, no contact, the characteristics of automation. This unit use this technology successfully on developing infinite keys automatic assembly equipment, for an enterprise, the equipment is used for infinite keys ontology and keys, automatic latch equipped , automatic detection and preventing leakage and key assembly back function.

\section{Machine vision principle}

Machine vision is to use robots to replace human eye and brain to do measurement and judgment. Machine vision system through optical device and non contact image sensor (namely image extraction device, CMOS and CCD ) 1 get images of the target object,. Then it will be taking based on image pixel distribution and brightness, color and other information of the target, convert the image of target into digital image signal, and then sent to the special image processing system. The special image processing system use all kinds of processing on these signals to extract the characteristics of the target, processing analysis and measurement, the image feature vector, and make qualitative analysis and quantitative detection on the measurement results .Then according to the results of identification to automatically control the action of field devices. 
Machine vision detection system mainly includes lighting light source, lens optical system, photoelectric sensors, image acquisition, image processing system, and communication interface ${ }^{[1]}$. As shown in the figure below, Fig1

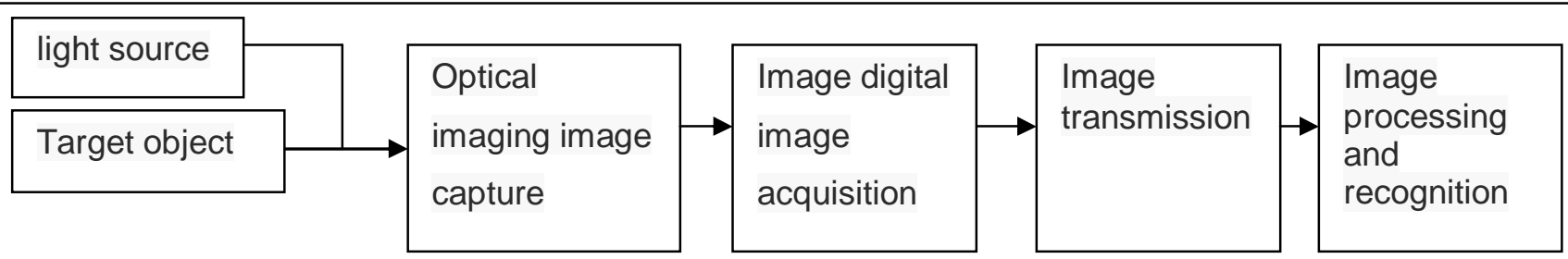

\section{A utomatic assembly equipment research and development of wireless key}

This paper studies the automatic assembly equipment of wireless key, wireless key ontology with the keys, bolt automatic assembly and automatic detection, preventing leakage and key assembly back function ${ }^{[2]}$. The key is processing the the only character image on key body that can identificate of the key to implemrnt assembly. Image recognition process is as follows.

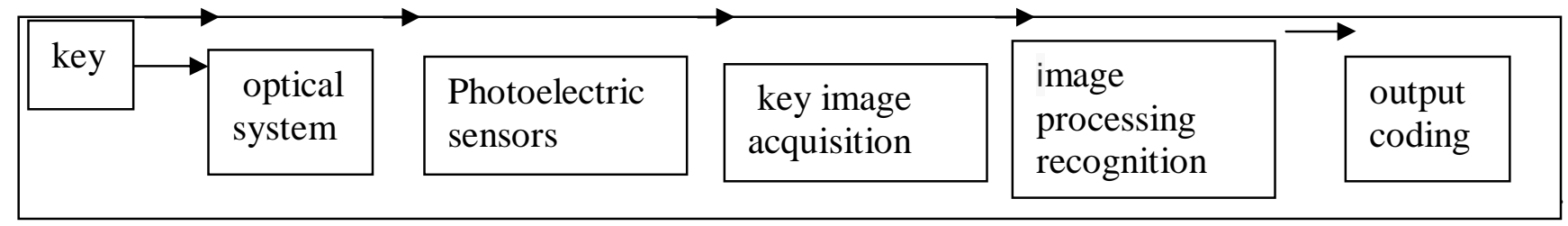

(2)Select digital image processor. Image processing is the core of the whole system, all the operation and control by the processor, as well as balancing the performance of the processor, at the same time also need to consider the development difficulty and workload of processor platform

(3)The image recognition algorithm. First, System will let the original signal of CDD image sensor output running the key characters which are geted through image recognition methodin the processor. The basic flow chart is as follows.

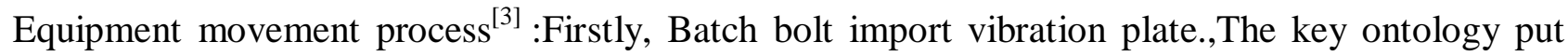
back in place Secondly, operators place the key piece on the copy milled. Then operators exit the grating region and hands start button. At this time, the key ontology is clamping. Thirdly, Automatically detects the key body is put in place and whether the correct. If testing is qualified, the placing rack of key ontology willb put forward to the assembly. If the detection is abnormal, it will appeare thecorresponding alarm. Then according to the alarm message resets the key ontology or keys. And then restart the hands button, The key ontology put frame automatically sent to the assembly. The key piece automatically installed. The fourth, the key piece automatically push offset fixed angle. Bolt automatically sent to the assembly, automatic assembly in place. The fifth, automatically detect whether bolt installed incorrectly or in place. If test is qualified, the key ontology is placed back to the pick-up. Then, operator remove key ontology. If the detection is abnormal, it will appeare the corresponding alarm. At this time, it need open fixture and take out the key assembly artificially. And then it need Repair processing. 


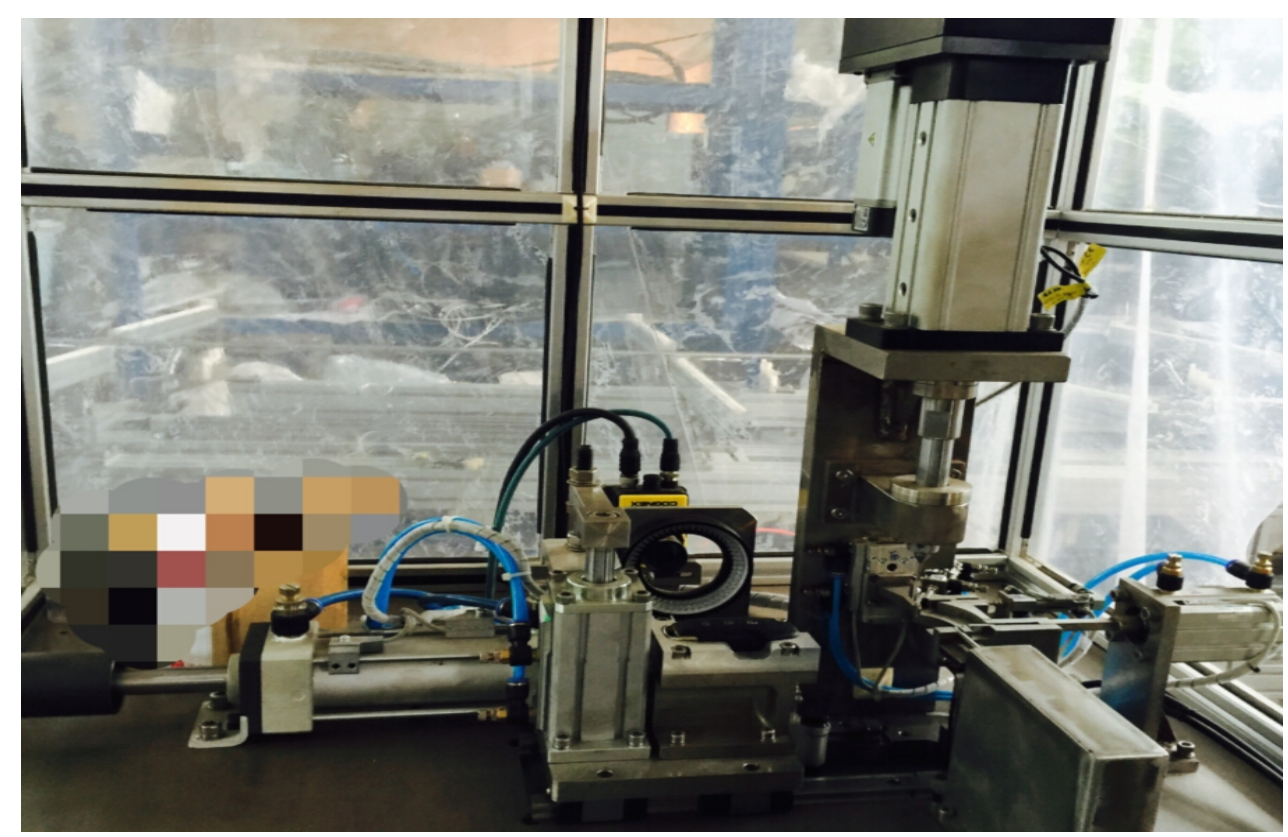

Fig.3 internal structure of key to automatic assembly equipment

\section{The key control points of wireless key automatic assembly equipment}

The equipment includes automatic assembly equipment and testing devices. Key ontology, bolt, and the key chip positioning is reliable. Place should ensure convenient smoothly. Wrong tooling need to ensure the product protection and can't touch scratch on product appearance. Key ontology and the key is a one-to-one relationship. We use manual placement on the tooling. And then it realize automatic assembly. Bolt use vibration plate automatic sorting and automatically installed. And it need reserve the function of manually placed the bolt. Intake capacity of Bolt vibration plate is not less than 2000. And it has alarm function of lack of material. Bolt installation should be oriented, to ensure that the bolt installed accurately. The key assembly bracket moving and key to offset have high repeat precision, ensure that every pin can accurate assembly in place. When the packing is detected, if you need to logoff repair, permissions to loosen the fixture body by passing the password. Inside the machine need domestic and lighting illumination requirement is more than $10001 \mathrm{x}^{[4]}$.

Wireless key automatic assembly equipment with machine vision photograph the only characters on the keys body. Images are stored on the device's own industrial. Photos at least more than 200000 . As the picture number achieve 200000, each new one automatically will cover the front of the picture $^{[5]}$

\section{conclusion}

In this paper, we study the wireless key equipment equipment. It has successfully applied in auto industry, realized the car keys assembly automation (assembly automation), improved the enterprise production efficiency, reduced cost and ensure the quality of products. It has great significance especially under special conditions to reducing labor intensity, or replace the manual assembly.

\section{Reference}

[1]Positioning technology based on image sequences of robot vision research. Journal of Qingdao university. 2010 
[2] Christian Floerkemeier,Sanjay Sarma.An Overview of RFID System Interfaces and Reader Protocols. IEEE International Conference on RFID.2008

[3] NXP corperation.PCF7938XA Security Transponder (HT3) datasheet. . 2011

[4] Disc locks lock automatic assembly system research and development. HFUT.2011

[5] Based on the model development car keyless start controller design and implementation. SJTU.2013 